\title{
Pathophysiology of atrial fibrillation in endurance athletes: an overview of recent findings
}

\author{
Fabian Sanchis-Gomar MD PhD, Alejandro Lucia MD PhD
}

$\mathrm{E}$ xercise training in general has been associated with a reduced risk of atrial fibrillation and associated morbidity among patients with this condition. ${ }^{1-3}$ However, more strenuous endurance exercise (e.g., marathon running) appears to increase the risk of paroxysmal atrial fibrillation in otherwise healthy young or middle-aged athletes with no cardiac structural abnormality. ${ }^{4}$ Atrial fibrillation is a common heart arrhythmia usually associated with advanced age, hypertension, diabetes and obesity as well as with structural cardiac changes. It often complicates heart failure or coronary ischemic disease. It is not yet known whether atrial fibrillation associated with strenuous exercise is mechanistically similar to atrial fibrillation occurring in nonathletic, older people or whether it is a different entity with different management requirements. We consider early evidence examining the potential mechanisms linking strenuous exercise to atrial fibrillation in young, otherwise normal hearts in an attempt to distinguish the idiosyncrasies of atrial fibrillation related to strenuous endurance exercise from that occurring in nonathletic older people.

Experts have proposed increased vagal tone and cholinergic stimulation (so-called vagal atrial fibrillation), atrial inflammation and fibrosis (cardiac auriculitis), remodelling and enlargement of the left atrium, and ectopy (particularly pulmonary vein ectopy) as triggers and modulators of atrial fibrillation associated with strenuous endurance exercise. ${ }^{5}$ Others have also suggested that some endurance athletes may experience extensive stiffening of the heart muscle, which might trigger atrial fribrillation at high-intensity exercise. ${ }^{6}$

A recently emphasized concept is that of vagal atrial fibrillation, ${ }^{7}$ which usually affects young patients with otherwise normal hearts. It remains an underrecognized entity, with no universal definition, cause or diagnostic criteria, but with some common features, namely atrioventricular block, asystolic periods, sinus bradycar- dia and increased variability in heart rate. Carpenter and colleagues ${ }^{7}$ have postulated that vagal stimuli such as eating, sleeping, relaxation following stress and exercise, and alcohol consumption are triggers. ${ }^{7}$ Exposure to these types of stimuli, coupled with withdrawal of sympathetic tone, may result in imbalanced stimulation of the sympathetic and parasympathetic systems, along with increased atrial ectopy and atrial substrate modification. The authors thus proposed vagal atrial fibrillation to explain the greater prevalence of paroxysmal atrial fibrillation among younger athletes. ${ }^{7}$ Recent mechanistic research with rats also supports the contribution of vagal promotion (particularly via increased baroreflex responsiveness and cardiomyocyte sensitivity to cholinergic stimulation) in atrial fibrillation induced by chronic endurance exercise, possibly due to downregulation of regulators of G protein-signalling (RGS) 4 function. ${ }^{8}$

Recent research has also identified the proinflammatory cytokine tumour necrosis factor- $\alpha$ (TNF- $\alpha$ ) as being important in the pathophysiology of the inflammation and fibrosis observed in atrial fibrillation associated with strenuous exercise. Indeed, an experimental study recently showed that six weeks of intense endurance training in mice increased vulnerability to atrial fibrillation in association with inflammation and fibrosis in the atrial tissue while inducing TNF- $\alpha$-dependent activation of both the nuclear factor kappa light-chain enhancer of activated B cells (NFKB) and p38 mitogen-activated protein kinase (MAPK). ${ }^{9}$ Conversely, TNF- $\alpha$ inhibition

\section{KEY POINTS}

- Strenuous endurance exercise appears to increase the risk of paroxysmal atrial fibrillation in otherwise healthy young or middleaged athletes.

- It remains unknown whether atrial fibrillation associated with strenuous endurance exercise is mechanistically similar to that occurring in nonathletic older people.

- Current evidence suggests that the pathophysiology and clinical consequences of atrial fibrillation in healthy athletes differ from those in older people with failing hearts.
Competing interests: None declared.

This article has been peer reviewed.

Correspondence to: Fabian Sanchis-Gomar, fabian.sanchis@uv.es

CMAJ 2016. DOI:10.1503 /cmaj.160416 
(by TNFA gene ablation or treatment with the biopharmaceutical etanercept used in TNF- $\alpha$ linked autoimmune inflammatory disorders) or p38 inhibition prevented strenuous-exerciseinduced atrial structural remodelling and susceptibility to atrial fibrillation without affecting beneficial adaptations from exercise (e.g., improved heart pump function and reduced heart rate).

MicroRNAs are small noncoding genome regions that regulate myriad cell processes and body functions, including cardiac function under physiologic and pathological conditions (e.g., they help reconstruct ion channels by regulating gene expression in cardiomyocytes during the process of arrhythmia). MicroRNAs are therefore potential biomarkers of atrial fibrillation that could help our understanding of the pathophysiology of the condition, including atrial fibrillation related to strenuous endurance exercise. Notably, microRNA-26 is downregulated in tissue of the right atrium in patients with atrial fibrillation, which causes pro-fibrillatory upregulation of the inward-rectifier potassium current and shortening of the action potential duration..$^{10}$ In addition, circulating microRNA-29b is downregulated in patients with heart failure and atrial fibrillation ${ }^{11}$ and was recently shown to be reduced in the blood of healthy trained runners after completing a marathon. ${ }^{12}$ Clauss and colleagues ${ }^{12}$ linked two cardiac tissue-specific microRNAs associated with myocardial ischemia-induced arrhythmia microRNA-1 and microRNA-133a - to the diameter of the left atrium in trained marathon runners. Both markers were upregulated and correlated with the diameter of the left atrium 24 hours after a marathon in well-trained athletes but not in runners with a lower-intensity training background. The authors hypothesized that the level of microRNA expression may explain, at least in part, the discrepancy between beneficial moderate exercise and potentially harmful strenuous endurance exercise, and that circulating microRNAs could serve as biomarkers of pro-arrhythmogenic signalling leading to atrial enlargement after long-term strenuous endurance exercise.

More mechanistic research is needed to unravel the physiopathology of atrial fibrillation associated with strenuous exercise and to determine whether, and how, this condition affects the structure of atrial tissue.

Currently, reduced exercise load, or at least reduced training volume, is recommended to minimize the burden of vagally mediated atrial fibrillation, although this approach is seldom acceptable to competitive athletes. However, reducing training volume may not be sufficient to prevent recurrence of atrial fibrillation in all cases. In the recent guideline from the American Heart Association and the American College of Cardiology on the eligibility and disqualification recommendations for competitive athletes with cardiovascular abnormalities, ${ }^{13}$ the management options for atrial fibrillation in athletes include rate control or rhythm control. However, rate control may not be ideal, at least for competitive athletes, because it impairs performance: the reduction in heart rate is accompanied by a decrease in cardiac output and thus a limited maximal aerobic capacity, as reflected by a lower maximum oxygen uptake. In addition, adequate rate control is inherently difficult to ensure during training and competition, in which maximum efforts are reached.

Other suggested options include the use of antiarrhythmic drugs such as flecainide and amiodarone for symptomatic benefits in the short term. The use of $\beta$-blockers, digoxin and propafenone is discouraged because these drugs could exacerbate vagal atrial fibrillation. ${ }^{7}$ Circumferential pulmonary vein ablation, to prevent progression to persistent or even permanent atrial fibrillation, appears the best option for athletes with severe symptoms or those determined to continue training for maximal sports performance. ${ }^{13}$ Future research might determine whether anti-inflammatory treatment in athletes could be useful based on findings in animal research. ${ }^{9}$

Early evidence suggests that the pathophysiology of atrial fibrillation in otherwise healthy athletes engaging in strenuous endurance exercise may differ from that of atrial fibrillation commonly seen in older nonathletic people with failing hearts. More research is needed to develop effective treatment approaches for this "new" clinical entity. Furthermore, cardiac auriculitis has not yet been well-studied and deserves special investigation.

\section{References}

1. Reed JL, Birnie DH, Pipe AL. Exercise training in patients with paroxysmal, persistent or permanent atrial fibrillation. CMAJ 2014;186:E558.

2. Malmo V, Nes BM, Amundsen BH, et al. Aerobic interval training reduces the burden of atrial fibrillation in the short term: a randomized trial. Circulation 2016;133:466-73.

3. Santos-Lozano A, Sanchis-Gomar F, Barrero-Santalla S, et al. Exercise as an adjuvant therapy against chronic atrial fibrillation. Int J Cardiol 2016;207:180-4.

4. Abdulla J, Nielsen JR. Is the risk of atrial fibrillation higher in athletes than in the general population? A systematic review and meta-analysis. Europace 2009;11:1156-9.

5. D'Ascenzi F, Cameli M, Ciccone MM, et al. The controversial relationship between exercise and atrial fibrillation: clinical studies and pathophysiological mechanisms. J Cardiovasc Med (Hagerstown) 2015;16:802-10.

6. Leischik R, Spelsberg N, Niggemann H, et al. Exercise-induced arterial hypertension: An independent factor for hypertrophy and a ticking clock for cardiac fatigue or atrial fibrillation in athletes? F1000 Res 2014;3:105. 
7. Carpenter A, Frontera A, Bond R, et al. Vagal atrial fibrillation: What is it and should we treat it? Int J Cardiol 2015;201:415-21.

8. Guasch E, Benito B, Qi X, et al. Atrial fibrillation promotion by endurance exercise: demonstration and mechanistic exploration in an animal model. J Am Coll Cardiol 2013;62:68-77.

9. Aschar-Sobbi R, Izaddoustdar F, Korogyi AS, et al. Increased atrial arrhythmia susceptibility induced by intense endurance exercise in mice requires TNFalpha. Nat Commun 2015;6:6018.

10. Luo X, Pan Z, Shan H, et al. MicroRNA-26 governs profibrillatory inward-rectifier potassium current changes in atrial fibrillation. J Clin Invest 2013;123:1939-51.

11. Dawson K, Wakili R, Ordög B, et al. MicroRNA29: a mechanistic contributor and potential biomarker in atrial fibrillation. Circulation 2013;127:1466-75, 75e1-28.

12. Clauss S, Wakili R, Hildebrand B, et al. MicroRNAs as Biomarkers for Acute Atrial Remodeling in Marathon Runners (the miRathon Study - a sub-study of the Munich Marathon Study). PLoS One 2016;11:e148599.
13. Zipes DP, Link MS, Ackerman MJ, et al. Eligibility and disqualification recommendations for competitive athletes with cardiovascular abnormalities: Task Force 9: arrhythmias and conduction defects: a scientific statement from the American Heart Association and American College of Cardiology. Circulation 2015;132:e315-25.

Affiliations: Research Institute of the Hospital Universitario 12 de Octubre (Sanchis-Gomar, Lucia); European University of Madrid (Lucia), Madrid, Spain

Contributors: Both authors were involved in the conception of the manuscript and performed independent literature reviews. Fabian Sanchis-Gomar drafted the manuscript and Alejandro Lucia revised it critically for important intellectual content. Both authors approved the final version to be published and agreed to act as guarantors of the work. 